\title{
Should patients with haemoptysis and a normal chest X-ray be bronchoscoped?
}

\author{
R.W. Heaton \\ Department of Medicine, Charing Cross Hospital, Fulham Palace Road, London W6 8RF, UK.
}

\begin{abstract}
Summary: A review of bronchoscopic records over a 5 year period identified 41 patients who had undergone fibreoptic bronchoscopy after presenting with haemoptysis and a normal chest X-ray. Carcinoma of the bronchus was found in 4 patients $(9.7 \%)$ and the procedure yielded a diagnosis in 8 of the 20 patients in whom a specific cause of their bleeding could be identified. The diagnostic yield is sufficiently high and the morbidity of the procedure sufficiently low to justify the investigation in patients presenting with haemoptysis and a normal chest $X$-ray.
\end{abstract}

\section{Introduction}

Although the role of flexible fibreoptic bronchoscopy in the investigation of patients with haemoptysis and a mass lesion on chest X-ray is undisputed ${ }^{1,2}$ there is less agreement about the need for the procedure in patients with a normal chest X-ray. Some authors have reported a high incidence of bronchial carcinomas in such patients ${ }^{3}$ whereas others have found no tumours, and have suggested that bronchoscopy is unnecessary. ${ }^{4}$ Many of the reported series deal with early experience after the introduction of fibreoptic bronchoscopy, and there are few reports dealing with the findings in this category of patients as part of an established service procedure. We report the findings of a review of patients investigated between 1980 and 1985 for haemoptysis who had a normal chest X-ray. Followup data for a mean of 18 months is also presented.

\section{Methods}

The bronchoscopy records of Charing Cross Hospital were searched for the period from September 1980 to March 1985 to identify those patients undergoing the procedure for haemoptysis, who had a normal chest $\mathrm{X}$-ray at the time of the examination. X-rays were reviewed to confirm the absence of localizing abnormalities. Details of each case presentation, outcome and follow-up data were obtained from the clinical case notes. No attempt was made to divide patients into those having early bronchoscopy, at the time of the haemoptysis, and those in whom the examination was performed late, after the bleeding had subsided.

Fibreoptic bronchoscopy was performed under

Correspondence: R.W. Heaton M.A., M.R.C.P.

Accepted: 30 April 1987 local anaesthesia by consultant physicians and senior registrars. All bronchial segments were identified and examined for sources of bleeding. Specimens were obtained by washing for microbiology, including stain and culture for acid-fast bacilli; and for cytology, by brushing, with biopsy when suspicious lesions were seen.

\section{Results}

Forty-four patients were identified in whom bronchoscopy was performed because of haemoptysis with a normal chest $X$-ray. On review of all the available radiology, including follow-up films, it was felt that in three cases the X-ray was abnormal at the time of the procedure, and these patients were excluded from the analysis. Details of the 41 remaining patients are summarised in Table I.

A specific diagnosis was reached in 8 patients as a result of the initial bronchoscopy. Four patients, all smokers (aged 60-71), had a visible carcinoma of the bronchus. Three were seen to be bleeding from abnormalities in the nose or on the tongue. In one patient Mycobacterium xenopi was isolated from the bronchial washings, with subsequent confirmation from sputum specimens.

Additional investigations produced a diagnosis in a further 12 cases (Table II). Six patients were diagnosed by bronchography as having bronchiectasis. One nonsmoker had a further bout of haemoptysis 10 months after his initial investigation, and at that time a small lesion was seen peripherally in the right lower lobe, shown to be an adenocarcinoma on needle biopsy. In three patients there was a systemic cause for abnormal bleeding: chronic renal failure in two and chronic 
Table I Details of 41 patients reported

\begin{tabular}{lccc}
\hline & Smokers & Non-smokers & Total \\
\hline $\mathrm{n}$ & 27 & 14 & 41 \\
Mean age (range) years & $58.4(21-77)$ & $54.5(18-75)$ & $56.3(18-77)$ \\
$\mathrm{M}: \mathrm{F}$ (rangth of followup & $16.2(1-48)$ & $26.2(6-52)$ & $18.3(1-52)$ \\
Mean lenge months & & & \\
(range) & & \\
\hline
\end{tabular}

lymphatic leukaemia in the other. Two were subsequently felt to have had a haematemesis rather than a haemoptysis.

Nine patients had a clear history of a preceding respiratory infection, with production of purulent sputum, and in seven of these, no other diagnosis was reached. Nine patients were noticed to have 'bronchitis' or a generalized inflammation of the airways by the endoscopist. Twelve patients had documented chronic obstructive airways disease.

Six patients died during the period of follow-up one from a squamous cell carcinoma diagnosed at bronchoscopy when his chest X-ray was normal, after surviving 17 months. The other five died from nonrespiratory causes. Post-mortem examination in three revealed no undiagnosed cause of haemoptysis. Of the patients in whom no specific diagnosis could be made, only one has had persistent and repeated complaints of haemoptysis.

\section{Discussion}

Carcinoma of the bronchus was diagnosed at bronchoscopy in 4 patients $(9.7 \%)$. Combining the current results with those of previous studies ${ }^{2-7}$ yields an overall incidence of carcinoma of $7.8 \%$ in patients undergoing bronchoscopy for haemoptysis with a normal chest X-ray (24 cases in 307 patients). Zavala ${ }^{3}$ reported an incidence as high as $16 \%$, whereas Solomon et al. ${ }^{6}$ and Heimer et al. ${ }^{4}$ found no cancers, but in the latter study only seven of the patients were smokers over the age of 45 years.

A final diagnosis of bronchiectasis was made in six patients (14.6\%), all of whom were chronic sputum producers. This compares with the $15 \%$ incidence reported by Jones et al. ${ }^{9}$ who specifically studied the role of bronchography in the investigation of patients with haemoptysis and a normal chest X-ray. Their suggestion that bronchoscopy be combined with bronchography in the investigation of such patients would be supported by the current results.

One further carcinoma was diagnosed during the follow-up period in a patient whose symptoms recurred. Adelman et al. ${ }^{8}$ found one carcinoma during follow-up of 67 patients with an initially normal bronchoscopy, and Jones et al. ${ }^{9}$ reported two peri pheral carcinomas becoming evident during follow-ue of 96 patients.

A specific diagnosis was reached in 20 of the 4 patients $(48.8 \%)$ reported here. Of the remaining 21 patients, only one has persistently complained of blood-stained sputum, and this has not been confirmed objectively, during a follow-up period up to 52 months post-bronchoscopy. In none of these patients has serious pulmonary pathology subsequently declared itself, over a mean follow-up period of 20 months. Adelman et al. ${ }^{8}$ described a similar resolution of symptoms and benign outcome in a group of 33 patients followed-up for 38 months post-bronchoscopy.

Given the low morbidity of fibreoptic bronchoscopy ${ }^{10}$ we would suggest that the incidence of carcinoma amongst these patients is sufficiently high to

Table II Specific diagnoses reached in 20 patients

\begin{tabular}{lcccc}
\hline & Total & Smokers & Non-smokers & Diagnosed at bronchoscopy \\
\hline Bronchiectasis & 6 & 3 & 3 & 4 \\
Carcinoma of the bronchus & 5 & 4 & 1 & 3 \\
Bleeding from upper airway & 3 & 2 & 1 & 1 \\
$\begin{array}{l}\text { Systemic cause of bleeding } \\
\text { chronic renal failure } \\
\text { chronic lymphocytic leukaemia }\end{array}$ & 3 & 2 & & 1 \\
Bleeding from gastrointestinal tract & 2 & & \\
Mycobacterium xenopi infection & 2 & 1 & 1 & 1 \\
\hline
\end{tabular}


warrant the examination, combined with bronchography through the bronchoscope if there is a clinical suspicion of bronchiectasis. Although carcinoma was diagnosed only in patients over the age of 60 in this series, bronchoscopy did reveal the cause of the bleeding in 3 of the 6 patients who were younger than 40 years, and one further patient in this age group was subsequently found to have bronchiectasis. The

\section{References}

1. Weaver, L.J., Solliday, N. \& Cugell, D.W. Selection of patients with haemoptysis for fibre-optic bronchoscopy. Chest 1979, 76: 7-10.

2. Mitchell, D.M., Emerson, C.J., Collyer, J. \& Collins, J.V. Fibre-optic bronchoscopy: ten years on. Br Med J 1980, 281: $360-363$.

3. Zavala, D.C. Diagnostic fibre-optic bronchoscopy: techniques and results of biopsy in 600 patients. Chest 1975, 68: $12-19$.

4. Heimer, D., Bar-Ziv, J. \& Scharf, S.M. Fibre-optic bronchoscopy in patients with haemoptysis and non-localising chest roentgenograms. Arch Intern Med 1985, 145: $1427-1428$.

5. Gong, H. \& Salvatierra, C. Clinical efficacy of early and delayed fibre-optic bronchoscopy in patients with haemoptyses. Am Rev Respir Dis 1981, 124: 221-225.

6. Solomon, D.A., Solliday, N.H. \& Gracey, D.R. Cytology in fibre-optic bronchoscopy. Comparison of recommendation that bronchography be carried out in all such patients ${ }^{9}$ is clearly impractical in most hospitals. Where bronchoscopy, combined if indicated with bronchography, is entirely normal, patients may be reassured that their haemoptysis is likely to be selflimiting and non-recurrent, with little likelihood of serious underlying pathology, and that prolonged follow-up is unnecessary.

bronchial brushing, washing and post-bronchoscopy sputum. Chest 1974, 65: 616-619.

7. Rath, G.S., Schaff, J.T. \& Snider, G.L. Flexible fibreoptic bronchoscopy. Techniques and review of 100 bronchoscopies. Chest 1973, 63: 689-693.

8. Adelman, M., Haponik, E.F., Bleecker, E.R. \& Britt, E.J. Cryptogenic haemoptysis. Clinical features, bronchoscopic findings and natural history in 67 patients. Ann Intern Med 1985, 102: 829-834.

9. Jones, D.K., Cavanagh, P., Schneerson, J.M. \& Flower, C.D.R. Does bronchography have a role in the assessment of patients with haemoptysis? Thorax 1985, 40: 668-670.

10. Simpson, F.G., Arnold, A.G., Purvis, A., Belfield, P.W., Muers, M.F. \& Cooke, N.J. Postal survey of bronchoscopic practise by physicians in the United Kingdom. Thorax 1986, 41: 311-317. 